\title{
Association between Hyperhomocysteinemia and Thyroid Hormones in Euthyroid Diabetic Subjects
}

\author{
Yun Zhang, Qiong Wang, Quanzhong Li, and Ping Lu \\ Department of Endocrinology, Henan Province People's Hospital and Zhengzhou University People's Hospital, \\ 7 Weiwu Road, Zhengzhou, Henan 470003, China
}

Correspondence should be addressed to Ping Lu; luping.henan@gmail.com

Received 19 March 2015; Revised 21 May 2015; Accepted 11 June 2015

Academic Editor: Betti Giusti

Copyright (C) 2015 Yun Zhang et al. This is an open access article distributed under the Creative Commons Attribution License, which permits unrestricted use, distribution, and reproduction in any medium, provided the original work is properly cited.

Objectives. The concept now emerging is that higher thyroid-stimulating hormone (TSH) and lower thyroid hormone levels within the euthyroid range may adversely affect atherosclerosis. The present study aimed to investigate the potential associations between thyroid parameters and hyperhomocysteinaemia in a cohort of euthyroid diabetic subjects. Material and Methods. Two hundred and seventy-three euthyroid diabetic subjects (167 males and 106 females) were consecutively recruited in this cross-sectional study. Clinical and biomedical data was collected. Results. TSH level was higher in females than males. Compared to normal-homocysteine group, hyperhomocysteinaemia group was more likely to be elderly, males, with longer diabetes history, and with lower diastolic blood pressure. Free thyroxine (FT4) level was lower in hyperhomocysteinaemia group than in normal-homocysteine group; however, it was not statistically significant. Adjusted for age, sex, body mass index, duration of diabetes, blood pressure, fasting glucose, total cholesterol, and triglyceride in logistic regression analyses, hyperhomocysteinaemia was significantly correlated with FT4 $(P=0.021)$. No significant association was found with TSH or free triiodothyronine. When analyzed in subjects with TSH $<$ $2.5 \mathrm{uIU} / \mathrm{mL}$ separately, we got similar results. Conclusions. In conclusion, we identified a relation between hyperhomocysteinemia and FT4 in a group of euthyroid diabetic patients.

\section{Introduction}

Thyroid dysfunction is a risk factor for cardiovascular disease, yet most subjects at risk for cardiovascular disease are euthyroid in the clinical setting. The concept now emerging is that higher thyroid-stimulating hormone (TSH) and lower thyroid hormone levels within the euthyroid range might adversely affect (subclinical) atherosclerosis [1-3]. Studies have revealed that free thyroxine (FT4), free triiodothyronine (FT3), and TSH are significantly associated with lipids profile in the euthyroid population regardless of gender [4-6]. However, studies in hypothyroid individuals show that elevated cholesterol and lipoprotein levels could not fully explain the increased cardiovascular morbidity. Other pathogenic factors might be involved.

Homocysteine is a sulfur-containing amino acid naturally found in human blood, which could induce endothelial injury, oxidative stress, smooth muscle hypertrophy and oxidation of low density lipoprotein cholesterol, and the process of atherosclerosis and cardiovascular diseases [7]. It has often been shown to be related to occlusive vascular disease independent of other known risk factors [8]. An earlier study indicated that an increase in plasma homocysteine level of $4 \mu \mathrm{mol} / \mathrm{L}$ conferred a $40 \%$ increase in relative risk for coronary heart disease compared with healthy controls [9]. Hyperhomocysteinemia in overt hypothyroidism had been found in many studies $[10,11]$. Even, in subclinical hypothyroidism patients, homocysteine concentration was reported to be higher as compared with euthyroid controls [12]. Furthermore, homocysteine level had been reported to be associated with carotid intima media thickness in patients with clinical hypothyroidism [13].

Diabetes mellitus, in particular type 2 diabetes, is also known to dramatically increase risk of cardiovascular diseases [14]. Thyroid disease and diabetes are the two most common endocrinopathies in the clinical practice, and they often coexist. Thyroid dysfunctions are more frequent in diabetic patients than in the general population [15]. Thyroid 
TABLE 1: Clinical characteristics of the subjects.

\begin{tabular}{|c|c|c|c|c|}
\hline & Total & Male & Female & $P$ \\
\hline$n$ & 292 & 167 & 106 & \\
\hline Age (years) & $54.50 \pm 12.03$ & $53.57 \pm 12.48$ & $55.96 \pm 11.20$ & 0.110 \\
\hline Duration of diabetes (years) & $8.35 \pm 7.31$ & $8.82 \pm 7.60$ & $7.62 \pm 6.81$ & 0.185 \\
\hline $\operatorname{BMI}\left(\mathrm{kg} / \mathrm{m}^{2}\right)$ & $25.23 \pm 3.69$ & $25.44 \pm 3.15$ & $24.90 \pm 4.40$ & 0.239 \\
\hline $\mathrm{SBP}(\mathrm{mmHg})$ & $134.48 \pm 16.09$ & $135.01 \pm 16.15$ & $133.64 \pm 16.03$ & 0.494 \\
\hline $\mathrm{DBP}(\mathrm{mmHg})$ & $82.92 \pm 9.85$ & $84.46 \pm 10.27$ & $80.49 \pm 8.66$ & 0.001 \\
\hline $\mathrm{TC}(\mathrm{mmol} / \mathrm{L})$ & $4.79 \pm 1.07$ & $4.76 \pm 1.09$ & $4.84 \pm 1.03$ & 0.524 \\
\hline $\mathrm{TG}(\mathrm{mmol} / \mathrm{L})$ & $1.99 \pm 1.77$ & $2.18 \pm 2.06$ & $1.70 \pm 1.14$ & 0.029 \\
\hline FBG (mmol/L) & $8.78 \pm 3.81$ & $8.88 \pm 3.91$ & $8.63 \pm 3.66$ & 0.607 \\
\hline Homocysteine $(\mu \mathrm{mol} / \mathrm{L})$ & $14.73 \pm 8.67$ & $16.23 \pm 10.00$ & $12.36 \pm 5.21$ & 0.000 \\
\hline FT3 (pmol/L) & $4.48 \pm 0.52$ & $4.61 \pm 0.55$ & $4.27 \pm 0.40$ & 0.000 \\
\hline FT4 (pmol/L) & $16.33 \pm 2.36$ & $16.44 \pm 2.31$ & $16.16 \pm 2.45$ & 0.346 \\
\hline TSH (uIU/mL) & $2.15 \pm 0.96$ & $2.05 \pm 0.93$ & $2.30 \pm 1.00$ & 0.037 \\
\hline
\end{tabular}

dysfunction had been associated with unfavorable changes in several metabolic parameters like lipid profile, which might amplify the cardiovascular disease risk in type 2 diabetes. Moreover, the absolute homocysteine level was also found to be higher in diabetic patients $[16,17]$.

Taking into account the considerations mentioned above, we hypothesized that effects of low thyroid function on increase of homocysteine level might extend into the euthyroid range, especially in diabetic individuals. The aim of this study was to investigate the potential associations between TSH and thyroid hormone levels within the normal range and hyperhomocysteinaemia in a cohort of euthyroid type 2 diabetic subjects.

\section{Materials and Methods}

2.1. Patients. Two hundred and seventy-three euthyroid type 2 diabetes subjects (167 males and 106 females) were consecutively recruited at inpatient clinic of Department of Endocrinology, Henan Province People's Hospital. Type 2 diabetes was diagnosed according to ADA 2009 criteria [18]. Euthyroidism was defined as TSH, FT3, and FT4 levels within their normal reference ranges (see the next paragraph). Exclusion criteria included type 1 diabetes, latent immune diabetes of the adults, gestational diabetes, and other types of diabetes [18], pregnancy, neoplasms, and any major medical condition in the 6 months preceding the study (i.e., liver, kidney, and heart failure). In particular, subjects with a previous history of thyroid diseases, such as overt hyper/hypothyroidism and thyroid cancer, were excluded. Subjects taking medications affecting thyroid hormone levels (such as thyroid supplementation and antithyroid agents, IFNc, amiodarone, lithium, corticosteroids, etc.) were also excluded. Informed consent was obtained from all participants. The present study was approved with the Institutional Ethnics Committee in Zhengzhou University.

2.2. Clinical, Anthropometric, and Laboratory Measurements. Clinical data was collected from all participants. Weight and height were measured with the subjects wearing light clothing and no shoes; the body mass index (BMI) was also calculated $\left(\mathrm{kg} / \mathrm{m}^{2}\right)$. Blood pressure was measured twice in the sitting position on the right arm using a sphygmomanometer (Erka), after at least 5 min of rest. Whole blood samples were drawn in the morning after an overnight fasting for the measurement of serum TSH, FT3, and FT4 levels, as well as biochemical indicators. Serum TSH, FT3, and FT4 levels were measured using chemiluminescence tests (Siemens Advia Centaur XP). The normal ranges were as follows: FT3 3.5-6.5 pmol/L; FT4 11.5-22.7 pmol/L; and TSH 0.55$4.78 \mathrm{uIU} / \mathrm{mL}$. Homocysteine was assayed using enzymatic methods (Roche Diagnostics), with reference values for adult male and females between 5 and $15 \mu \mathrm{mol} / \mathrm{L}$, together with fasting blood glucose (FBG), total cholesterol (TC), and triglyceride (TG).

2.3. Statistical Analyses. The data analysis was performed using SPSS version 18.0. All data was expressed as means \pm standard deviation (SD). All the subjects were divided into two groups according to homocysteine level: hyperhomocysteinaemia group $(>15 \mu \mathrm{mol} / \mathrm{L})$ and normal-homocysteine group $(\leq 15 \mu \mathrm{mol} / \mathrm{L})$. Two-sided $t$-tests and Pearson's Chisquare tests served to analyze the differences in means and proportions between the two groups. Logistic regression analysis was performed to evaluate the association of hyperhomocysteinaemia with thyroid parameters, adjusted for age, sex, BMI, duration of diabetes, systolic blood pressure (SBP), diastolic blood pressure (DBP), FBG, TC, and TG. Furthermore, subjects with TSH $<2.5 \mathrm{uIU} / \mathrm{mL}$ were analyzed separately. And statistical significance was defined as $P<$ 0.05 .

\section{Results and Discussion}

Table 1 showed the clinical characteristics of the 273 subjects. The mean age was $54.50 \pm 12.03$ years (range 23 to 82 ), and the mean BMI was $25.23 \pm 3.69 \mathrm{~kg} / \mathrm{m}^{2}$ (range 17.5 to 43.0 ). 
TABLE 2: Correlation of thyroid parameters with hyperhomocysteinaemia.

\begin{tabular}{|c|c|c|c|c|}
\hline & Hyperhomocysteinaemia & Normal-homocysteine & $P$ & $P^{\prime}$ \\
\hline$n$ & 100 & 173 & & \\
\hline Homocysteine $(\mu \mathrm{mol} / \mathrm{L})$ & $21.59 \pm 11.02$ & $10.76 \pm 2.38$ & 0.000 & \\
\hline Age (years) & $57.87 \pm 12.81$ & $52.55 \pm 11.14$ & 0.001 & 0.002 \\
\hline Sex (males/females) & $78 / 22$ & $89 / 84$ & 0.000 & 0.000 \\
\hline Duration of diabetes (years) & $9.60 \pm 7.86$ & $7.63 \pm 6.89$ & 0.039 & 0.678 \\
\hline BMI $\left(\mathrm{kg} / \mathrm{m}^{2}\right)$ & $24.92 \pm 3.20$ & $25.41 \pm 3.94$ & 0.286 & 0.221 \\
\hline SBP (mmHg) & $134.25 \pm 17.29$ & $134.61 \pm 15.40$ & 0.858 & 0.777 \\
\hline $\mathrm{DBP}(\mathrm{mmHg})$ & $81.08 \pm 10.91$ & $83.98 \pm 9.05$ & 0.019 & 0.015 \\
\hline $\mathrm{TC}(\mathrm{mmol} / \mathrm{L})$ & $4.80 \pm 1.15$ & $4.79 \pm 1.02$ & 0.931 & 0.907 \\
\hline $\mathrm{TG}(\mathrm{mmol} / \mathrm{L})$ & $2.19 \pm 2.40$ & $1.88 \pm 1.27$ & 0.224 & 0.035 \\
\hline $\mathrm{FBG}(\mathrm{mmol} / \mathrm{L})$ & $8.39 \pm 3.28$ & $9.00 \pm 4.08$ & 0.196 & 0.115 \\
\hline FT3 (pmol/L) & $4.49 \pm 0.59$ & $4.47 \pm 0.49$ & 0.785 & 0.736 \\
\hline FT4 (pmol/L) & $16.15 \pm 2.42$ & $16.64 \pm 2.24$ & 0.098 & 0.021 \\
\hline TSH (uIU/mL) & $2.23 \pm 1.02$ & $2.10 \pm 0.93$ & 0.260 & 0.061 \\
\hline
\end{tabular}

$P$ : differences between the two groups by Student's $t$-tests.

$P^{\prime}$ : association with hyperhomocysteinaemia by logistic regression analyses and with homocysteine level $(1=$ hyperhomocysteinaemia, $0=$ normalhomocysteine) as dependent variables and independent variables as follows: age, sex, BMI, duration of diabetes, BMI, SBP, DBP, TC, TG, FBG, FT3, FT4, and TSH.

Homocysteine, DBP, TG, and FT3 levels were lower in females than in males, and TSH levels were higher. No difference was found in age, BMI, FBG, FT4, or duration of diabetes (Table 1).

Compared to the normal-homocysteine group, the hyperhomocysteinaemia group was more likely to be elderly, males and have longer diabetes history and lower DBP levels. FT4 level was lower in hyperhomocysteinaemia group than in the normal-homocysteine group; however, it was not statistically significant. Adjusted for age, sex, BMI, duration of diabetes, blood pressure, FBG, TC, and TG in logistic regression analyses, hyperhomocysteinaemia was significantly correlated with FT4 $(P=0.021)$, age $(P=0.002)$, sex $(P=0.000)$, DBP $(P=0.015)$, and TG $(P=0.035)$. No significant association was found with FT3 $(P=0.736)$ or TSH $(P=0.061)$ (Table 2).

In 2003, the National Academy of Clinical Biochemistry (NACB) recommended lowering the upper reference limit of TSH to $2.5 \mathrm{uIU} / \mathrm{mL}$ based on a large-scale epidemiological survey that revealed that more than $95 \%$ of normal individuals had TSH levels $<2.5 \mathrm{uIU} / \mathrm{mL}$ and that those with higher TSH levels were likely to have various thyroid disorders [19]. So we did an analysis in subjects with TSH $<2.5 \mathrm{uIU} / \mathrm{mL}$ separately and got similar results: hyperhomocysteinaemia was still correlated with FT4 $(P=0.049)$ in logistic regression analysis (Table 3 ).

So, in this study, we demonstrated an association between hyperhomocysteinaemia, an independent risk factor of cardiovascular diseases, and lower FT4 levels, indicative of low normal thyroid function, in a group of euthyroid diabetic patients. No association was found with FT3 or TSH. Even when TSH was restricted to smaller than $2.5 \mathrm{uIU} / \mathrm{mL}$, we got similar results. The relationship between thyroid hormones and atherosclerosis in the euthyroid population has garnered much interest recently. These observations were in line with previous studies in overt hypothyroid patients. Study by Diekman et al. indicated that FT4 was an independent determinant of homocysteine concentrations; log (FT4) levels and age accounted for $28 \%$ of the variability of homocysteine [20]. Bamashmoos et al. reported that homocysteine was significantly positively correlated with TSH and negatively correlated with FT4 and there were no significant correlations with FT3 [21]. However, another study reported that homocysteine significantly negatively correlated only with FT3 [22].

Thyroid hormones were recognized as catabolic hormones and they regulated various processes of metabolism. Homocysteine metabolism might be influenced by thyroid hormone through two different pathways. Firstly, thyroid hormone could influence the activity of hepatic enzymes involved in the remethylation pathway of homocysteine, methionine synthase, and methylenetetrahydrofolate reductase [23-25]. Secondly, low level of thyroid hormone probably reduces glomerular filtration rate leading to increased creatinine and homocysteine levels [20, 26, 27]. Our study suggested that, even in euthyroid subjects with normal thyroid hormones and TSH, thyroid function might influence the homocysteine metabolism, leading to hyperhomocysteinaemia. And we could suppose that homocysteine might be involved in the association between thyroid function and atherosclerosis, even in euthyroid subjects.

Several methodological aspects and limitations of our study need to be considered. First of all, the causal relationship could not be inferred from this study because it was cross-sectional in nature. Secondly, only FBG level was used to represent glucose control, which was not good enough; however, glycated hemoglobin was not measured. Furthermore, in type 2 diabetes patients, homocysteine levels might be influenced by other variables, such as serum creatinine levels, comorbidities, and/or hypoglycemic therapies. 
TABLE 3: Correlation with hyperhomocysteinaemia in patients with $\mathrm{TSH}<2.5 \mathrm{uIU} / \mathrm{mL}$.

\begin{tabular}{|c|c|c|c|c|}
\hline & Hyperhomocysteinaemia & Normal-homocysteine & $P$ & $P^{\prime}$ \\
\hline$n$ & 69 & 124 & & \\
\hline Homocysteine $(\mu \mathrm{mol} / \mathrm{L})$ & $21.59 \pm 11.62$ & $10.78 \pm 2.41$ & 0.000 & \\
\hline Age (years) & $57.93 \pm 13.09$ & $51.35 \pm 10.43$ & 0.000 & 0.003 \\
\hline Sex (males/females) & $57 / 12$ & $68 / 56$ & 0.000 & 0.000 \\
\hline Duration of diabetes (years) & $10.01 \pm 8.62$ & $7.08 \pm 6.61$ & 0.016 & 0.586 \\
\hline $\operatorname{BMI}\left(\mathrm{kg} / \mathrm{m}^{2}\right)$ & $25.00 \pm 3.22$ & $25.56 \pm 3.98$ & 0.318 & 0.393 \\
\hline $\mathrm{SBP}(\mathrm{mmHg})$ & $134.35 \pm 18.54$ & $134.44 \pm 15.56$ & 0.972 & 0.747 \\
\hline $\mathrm{DBP}(\mathrm{mmHg})$ & $80.72 \pm 11.05$ & $83.66 \pm 9.37$ & 0.052 & 0.131 \\
\hline TC (mmol/L) & $4.78 \pm 1.07$ & $4.71 \pm 0.97$ & 0.634 & 0.334 \\
\hline TG $(\mathrm{mmol} / \mathrm{L})$ & $2.09 \pm 2.02$ & $2.04 \pm 1.36$ & 0.842 & 0.518 \\
\hline $\mathrm{FBG}(\mathrm{mmol} / \mathrm{L})$ & $8.21 \pm 3.00$ & $9.29 \pm 4.29$ & 0.041 & 0.063 \\
\hline FT3 (pmol/L) & $4.55 \pm 0.62$ & $4.49 \pm 0.47$ & 0.508 & 0.822 \\
\hline FT4 (pmol/L) & $16.28 \pm 2.42$ & $16.91 \pm 2.12$ & 0.060 & 0.049 \\
\hline TSH (uIU/mL) & $1.64 \pm 0.47$ & $1.61 \pm 0.50$ & 0.678 & 0.850 \\
\hline
\end{tabular}

$P$ : differences between the two groups by Student's $t$-tests.

$P^{\prime}$ : association with hyperhomocysteinaemia by logistic regression analyses and with homocysteine level $(1=$ hyperhomocysteinaemia, $0=$ normalhomocysteine) as dependent variables and independent variables as follows: age, sex, BMI, duration of diabetes, BMI, SBP, DBP, TC, TG, FBG, FT3, FT4, and TSH.

However, all these were not considered in this study. Also, as only 273 subjects were recruited, the statistical power of the present study was limited. Further investigations are needed to understand the association of homocysteine levels with thyroid parameters and the intimate mechanisms.

\section{Conclusions}

In conclusion, we identified an association between hyperhomocysteinemia and FT4 in a group of euthyroid type 2 diabetes patients for the first time. A longitudinal study is needed to assess the effects of the variation in thyroid hormone levels within the euthyroid range in the development of hyperhomocysteinaemia.

\section{Conflict of Interests}

The authors declare that there is no conflict of interests regarding the publication of this paper.

\section{References}

[1] B. O. Åsvold, T. Bjøro, C. Platou, and L. J. Vatten, “Thyroid function and the risk of coronary heart disease: 12-year follow-up of the HUNT Study in Norway," Clinical Endocrinology, vol. 77, no. 6, pp. 911-917, 2012.

[2] R. P. F. Dullaart, R. de Vries, C. Roozendaal, A. C. M. Kobold, and W. J. Sluiter, "Carotid artery intima media thickness is inversely related to serum free thyroxine in euthyroid subjects," Clinical Endocrinology, vol. 67, no. 5, pp. 668-673, 2007.

[3] N. Takamura, A. Akilzhanova, N. Hayashida et al., "Thyroid function is associated with carotid intima-media thickness in euthyroid subjects," Atherosclerosis, vol. 204, no. 2, pp. e77-e81, 2009.

[4] A. Roos, S. J. L. Bakker, T. P. Links, R. O. B. Gans, and B. H. R. Wolffenbuttel, "Thyroid function is associated with components of the metabolic syndrome in euthyroid subjects," The Journal of Clinical Endocrinology \& Metabolism, vol. 92, no. 2, pp. 491-496, 2007.

[5] J. D. J. Garduño-Garcia, U. Alvirde-Garcia, G. López-Carrasco et al., "TSH and free thyroxine concentrations are associated with differing metabolic markers in euthyroid subjects," European Journal of Endocrinology, vol. 163, no. 2, pp. 273-278, 2010.

[6] D. Pallas, D. A. Koutras, P. Adamopoulos, P. Marafelia, A. G. Souvatzoglou Piperingos, and S. D. Moulopoulos, "Increased mean serum thyrotropin in apparently euthyroid hypercholesterolemic patients: does it mean occult hypothyroidism?" Journal of Endocrinological Investigation, vol. 14, no. 9, pp. 743-746, 1991.

[7] M. Á. Medina, J. L. Urdiales, and M. I. Amores-Sánchez, "Roles of homocysteine in cell metabolism: old and new functions," European Journal of Biochemistry, vol. 268, no. 14, pp. 38713882, 2001.

[8] M. Den Heijer, S. Lewington, and R. Clarke, "Homocysteine, MTHFR and risk of venous thrombosis: a meta-analysis of published epidemiological studies," Journal of Thrombosis and Haemostasis, vol. 3, no. 2, pp. 292-299, 2005.

[9] C. J. Boushey, S. A. A. Beresford, G. S. Omenn, and A. G. Motulsky, "A quantitative assessment of plasma homocysteine as a risk factor for vascular disease: probable benefits of increasing folic acid intakes," Journal of the American Medical Association, vol. 274, no. 13, pp. 1049-1057, 1995.

[10] B. G. Nedrebø, U.-B. Ericsson, O. Nygård et al., "Plasma total homocysteine levels in hyperthyroid and hypothyroid patients," Metabolism: Clinical and Experimental, vol. 47, no. 1, pp. 89-93, 1998.

[11] M. S. Morris, A. G. Bostom, P. F. Jacques, J. Selhub, and I. H. Rosenberg, "Hyperhomocysteinemia and hypercholesterolemia associated with hypothyroidism in the third US National Health and Nutrition Examination Survey," Atherosclerosis, vol. 155, no. 1, pp. 195-200, 2001.

[12] E. Sengül, B. Cetinarslan, I. Tarkun, Z. Cantürk, and E. Türemen, "Homocysteine concentrations in subclinical 
hypothyroidism," Endocrine Research, vol. 30, no. 3, pp. 351-359, 2004.

[13] M. Gunduz, E. Gunduz, F. Kircelli, N. Okur, and M. Ozkaya, "Clinical study: role of surrogate markers of atherosclerosis in clinical and subclinical thyroidism," International Journal of Endocrinology, vol. 2012, Article ID 109797, 6 pages, 2012.

[14] P. Chamnan, R. K. Simmons, S. J. Sharp, S. J. Griffin, and N. J. Wareham, "Cardiovascular risk assessment scores for people with diabetes: a systematic review," Diabetologia, vol. 52, no. 10, pp. 2001-2014, 2009.

[15] R. Kadiyala, R. Peter, and O. E. Okosieme, “Thyroid dysfunction in patients with diabetes: clinical implications and screening strategies," International Journal of Clinical Practice, vol. 64, no. 8, pp. 1130-1139, 2010.

[16] T. Huang, J. Ren, J. Huang, and D. Li, "Association of homocysteine with type 2 diabetes: a meta-analysis implementing Mendelian randomization approach," BMC Genomics, vol. 14, no. 1, article 867, 2013.

[17] G. T. Russo, A. Giandalia, E. L. Romeo et al., "Lipid and nonlipid cardiovascular risk factors in postmenopausal type 2 diabetic women with and without coronary heart disease," Journal of Endocrinological Investigation, vol. 37, no. 3, pp. 261268, 2014.

[18] American Diabetes Association, "Standards of medical care in diabetes-2009," Diabetes Care, vol. 32, supplement 1, pp. S13S61, 2008.

[19] J. Kratzsch, G. M. Fiedler, A. Leichtle et al., "New reference intervals for thyrotropin and thyroid hormones based on national academy of clinical biochemistry criteria and regular ultrasonography of the thyroid," Clinical Chemistry, vol. 51, no. 8, pp. 1480-1486, 2005.

[20] M. J. M. Diekman, N. M. van der Put, H. J. Blom, J. G. P. Tijssen, and W. M. Wiersinga, "Determinants of changes in plasma homocysteine in hyperthyroidism and hypothyroidism," Clinical Endocrinology, vol. 54, no. 2, pp. 197-204, 2001.

[21] S. A. Bamashmoos, M. A. K. Al-Nuzaily, A. M. Al-Meeri, and F. H. H. Ali, "Relationship between total homocysteine, total cholesterol and creatinine levels in overt hypothyroid patients," SpringerPlus, vol. 2, article 423, 2013.

[22] A. Orzechowska-Pawilojc, M. Siekierska-Hellmann, A. Syrenicz, and K. Sworczak, "Homocysteine, folate, and cobalamin levels in hyperthyroid women before and after treatment," Endokrynologia Polska, vol. 60, no. 6, pp. 443-448, 2009.

[23] M. May-Sheng Chan and E. L. R. Stokstad, "Metabolic responses of folic acid and related compounds to thyroxine in rats," Biochimica et Biophysica Acta, vol. 632, no. 2, pp. 244-253, 1980.

[24] C. P. P. Nair, G. Viswanathan, and J. M. Noronha, "Folate-mediated incorporation of ring-2-carbon of histidine into nucleic acids: influence of thyroid hormone," Metabolism: Clinical and Experimental, vol. 43, no. 12, pp. 1575-1578, 1994.

[25] A. Ayav, J. M. Alberto, F. Barbe et al., "Defective remethylation of homocysteine is related to decreased synthesis of coenzymes B2 in thyroidectomized rats," Amino Acids, vol. 28, no. 1, pp. 3743, 2005.

[26] E. A. Lien, B. G. Nedrebø, J. E. Varhaug, O. Nygård, A. Aakvaag, and P. M. Ueland, "Plasma total homocysteine levels during short-term iatrogenic hypothyroidism," Journal of Clinical Endocrinology and Metabolism, vol. 85, no. 3, pp. 1049-1053, 2000.

[27] B. G. Nedrebø, O. Nygård, P. M. Ueland, and E. A. Lien, "Plasma total homocysteine in hyper- and hypothyroid patients before and during 12 months of treatment," Clinical Chemistry, vol. 47, no. 9, pp. 1738-1741, 2001. 


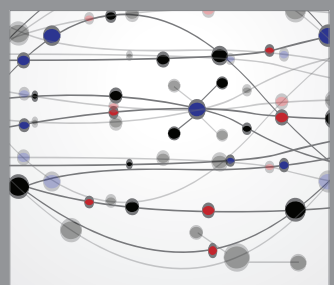

The Scientific World Journal
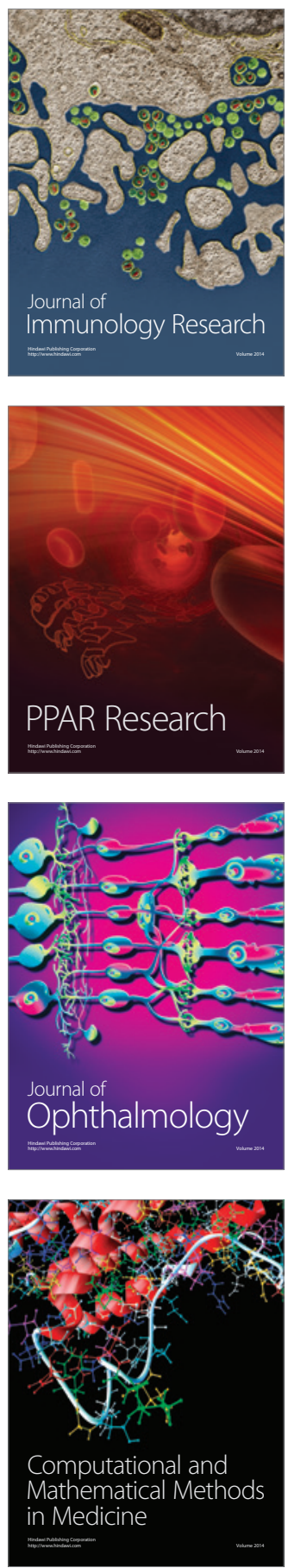

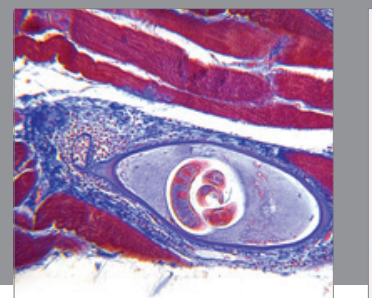

Gastroenterology

Research and Practice
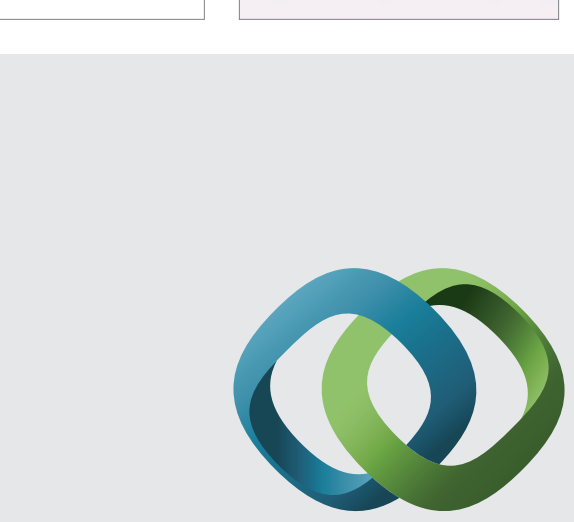

\section{Hindawi}

Submit your manuscripts at

http://www.hindawi.com
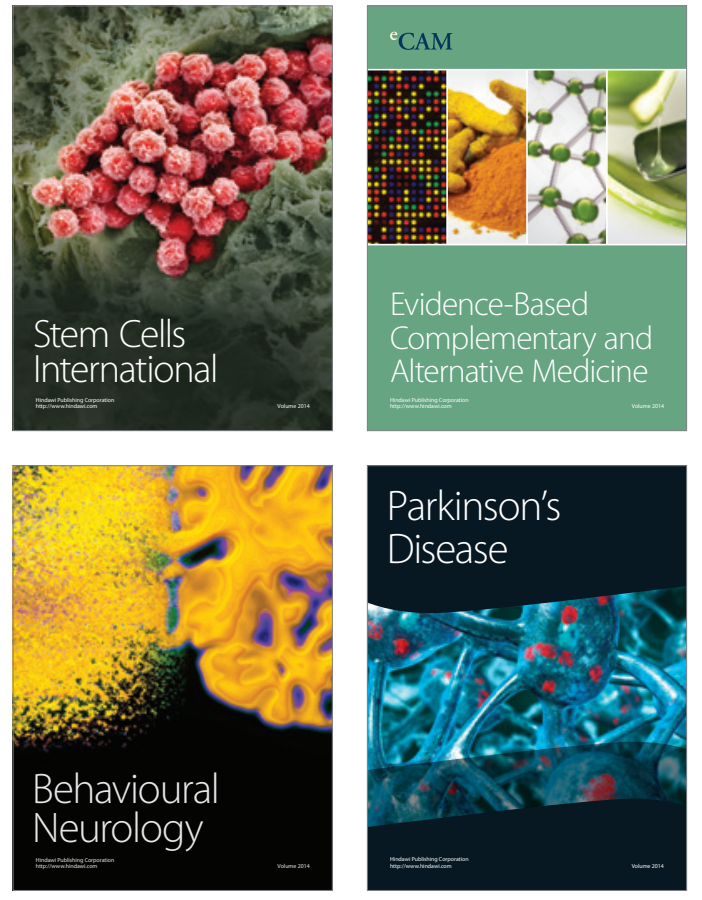
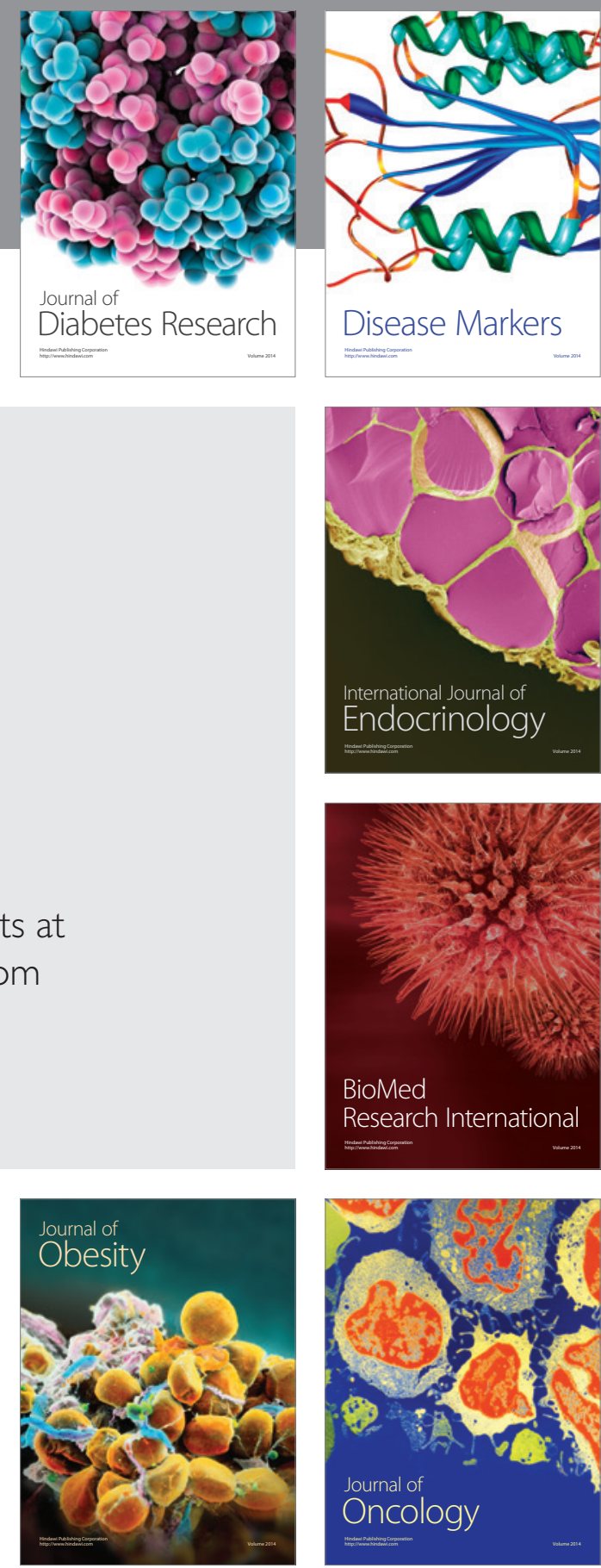

Disease Markers
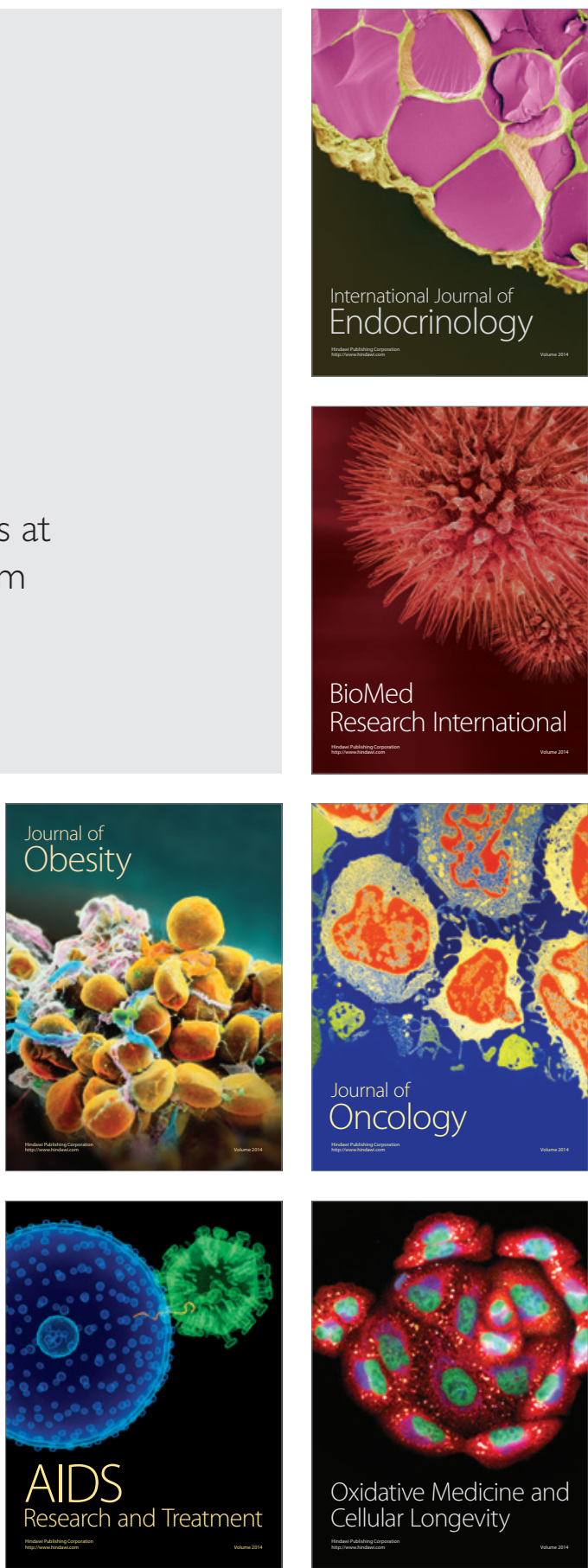